\title{
Control of Chaos Using the Controller Identification Technique
}

\author{
Alexandre Molter and Fabricio B. Cabral \\ Department of Mathematics and Statistics, Federal University of Pelotas, Campus Universitário, No. 354, 96010-900 Pelotas, RS, Brazil
}

Correspondence should be addressed to Alexandre Molter; alexandre.molter@yahoo.com.br

Received 10 July 2017; Revised 18 October 2017; Accepted 22 October 2017; Published 14 November 2017

Academic Editor: Jonathan N. Blakely

Copyright (c) 2017 Alexandre Molter and Fabricio B. Cabral. This is an open access article distributed under the Creative Commons Attribution License, which permits unrestricted use, distribution, and reproduction in any medium, provided the original work is properly cited.

\begin{abstract}
Modeling and simulation of chaotic system with dynamic control have been extensively presented in the past decades. Several control techniques have been proposed for the control of chaos. One technique that has not been sufficiently explored for the control of nonlinear systems is the controller identification technique. This technique is based on the evaluation of controllers even if they are not online. This technique does not use a priori knowledge of the plant parameters. In this work, we propose a class of controllers candidates to follow desired trajectories. Simulation results are presented for the control of chaotic systems.
\end{abstract}

\section{Introduction}

Phenomena which exhibit chaotic behavior appear in several areas, attracting researchers which try to describe this behavior through mathematical equations and to control its dynamic.

The search for efficient control techniques of nonlinear systems continues to be a challenge to researchers. Among a variety of controllers used in dynamical systems, proportional integrative derivative (PID) is the most common and practical to be adopted by control engineers. Since the PID controllers are commonly used in industrial control systems, they are usually adjusted by empirical methods. On the other hand, there are more sophisticated control techniques involving complex theoretical developments, which impose very restrictive hypothesis on the systems to be controlled, as nonlinear control techniques.

The theoretical success of robust control led many researchers to say that control should focus on the plant parameter estimation and its bounds. However, other researchers in the control area, such as Safonov and Tsao [1], become unsatisfied with the robust control paradigm, which required the control of a family of plants instead of the control of one, which would lead to conservative results. They stated that it was time to reformulate the control problem. A first formulation was the unfalsified control $[1,2]$. This control has two major characteristics: it advances from plant parameter estimation to controller parameter estimation [3] and it considers model falsification in the sense of Popper instead of model validation $[4,5]$. This was a first formulation of the controller identification problem, which in general considers a list of candidate controllers and a criterion to judge the performance of these controllers without needing to put them online [6].

In fact, there are few control techniques which require minimal information on the systems to be controlled. Among these techniques we can cite the ones based on the unfalsified control paradigm and the controller identification technique $[2,6]$. The unfalsified control paradigm allows us to formulate the control problem based on experimental data [7]. The advantage of this technique, when compared to others, is that it does not require a priori knowledge of the state or physical properties of the plant. This fact illustrates a potential to be explored through the use of the controller identification technique, which can also be used for the control of nonlinear dynamical systems.

The main goal of this work is to use the controller identification technique to control the trajectories of dynamical systems with chaotic behavior. The novelty of this approach is that the plant is treated as a model free plant which accounts for any model mismatches and that we try to identify a low order controller for a complex system.

The proposed technique will be applied to control a Rössler [8], a Lotka-Volterra three-species system [9-11], and 
a Rössler hyperchaos model [12]. In the literature we can find several works dealing with control of chaos. We highlight those related to the control of the Rössler system $[13,14]$ and the predator-prey systems [15-17]. In general, one can observe in these applications that the controllers used are of proportional kind. In the same way, in this work we use the controller identification technique applied to the proportional case. More precisely, given a class of candidate controllers, we identify the ones that present the best performance. The proportional control parameter is periodically modified. This update is made in order to put a better controller online. There are several ways to update the control parameters, the one chosen in this work is the step; that is, after a certain time, the control parameters are updated.

Numerical simulation is presented to illustrate the effectiveness of the proposed technique. These simulations regard the systems previously cited with the requirement of following a desired trajectory. The controllability of each system is calculated, which allows us to show that the proposed control technique preserves the locally complete state controllability of the nonlinear controlled systems.

This paper is organized as follows. In Section 2, the technique of controller identification is presented. In Section 3, a brief explanation of local controllability is presented and the proposed technique is applied to control the chaotic Rössler, predator-prey, and Rössler hyperchaos systems with simulations. In Section 4, some concluding remarks are given.

\section{The Controller Identification Technique}

A general overview of the controller identification technique can be found in [6]. According to this technique, the only plant information used is the plant experimental data. For the cases presented in this work, we need only the reference functions $r$ and the data $y$ (from the dynamical system) to obtain the control $u$. Given a desired behavior, a class of candidate controllers is proposed. Then, a controller is selected through the use of a performance index and the fictitious reference concept. In this work, we apply the controller identification to a class of proportional controllers and the respective mathematical development follows below.

The control law for a proportional controller is given by

$$
u=K(r-y)
$$

and, consequently, the fictitious reference is given by

$$
r_{K}=\frac{u}{K}+y
$$

where $K$ is a constant.

The performance index is given by

$$
I=\int_{0}^{t}\left(y-w_{m} * r_{K}\right)^{2} d \tau,
$$

where $W_{m}(s)$ is a transfer function of desired behavior and $w_{m}$ is its inverse Laplace transform

$$
w_{m}=\mathscr{L}^{-1}\left[W_{m}(s)\right] .
$$

Theorem 1. Among the controllers of class (1), the one that minimizes the performance index (3) is $\widehat{K}$ given by

$$
\widehat{K}=\frac{G}{F}
$$

where

$$
\begin{aligned}
F & =\int_{0}^{t}\left(y-y_{m}\right) u_{m} d \tau, \\
G & =\int_{0}^{t}\left(u_{m}\right)^{2} d \tau, \\
u_{m} & =w_{m} * u, \\
y_{m} & =w_{m} * y,
\end{aligned}
$$

with “*” denoting the convolution operation.

Proof. From (1), the fictitious reference is given by (2), where $K$ is a constant.

Using (8) and (9) we have that

$$
\begin{aligned}
y & -w_{m} * r_{K}=y-w_{m} *\left(\frac{u}{K}+y\right), \\
I= & \int_{0}^{t}\left(y-w_{m} * r_{K}\right)^{2} d \tau=\int_{0}^{t}\left(y-\frac{u_{m}}{K}-y_{m}\right)^{2} d \tau \\
& =\int_{0}^{t}\left(y^{2}+\left(\frac{u_{m}}{K}\right)^{2}+y_{m}^{2}-\frac{2 y u_{m}}{K}-2 y y_{m}\right. \\
& \left.+\frac{2 u_{m} y_{m}}{K}\right) d \tau=E-2 \frac{F}{K}+\frac{G}{K^{2}},
\end{aligned}
$$

where

$$
\begin{aligned}
& E=\int_{0}^{t}\left(y-y_{m}\right)^{2} d \tau, \\
& F=\int_{0}^{t}\left(y-y_{m}\right) u_{m} d \tau, \\
& G=\int_{0}^{t}\left(u_{m}\right)^{2} d \tau .
\end{aligned}
$$

The minimization of the performance index means finding $K$ that satisfies the equation:

$$
\frac{d I}{d K}=2 \frac{F}{K^{2}}-2 \frac{G}{K^{3}}=0,
$$

which leads to our estimator for the proportional constant

$$
\widehat{K}=\frac{G}{F} \text {. }
$$

One can note that $\widehat{K}=G / F$ is the multiplicative constant of control functions $u$. In order to obtain the simulation results, $\widehat{K}$ will be periodically updated. The objective is to minimize the difference between the measured $y$ and the 


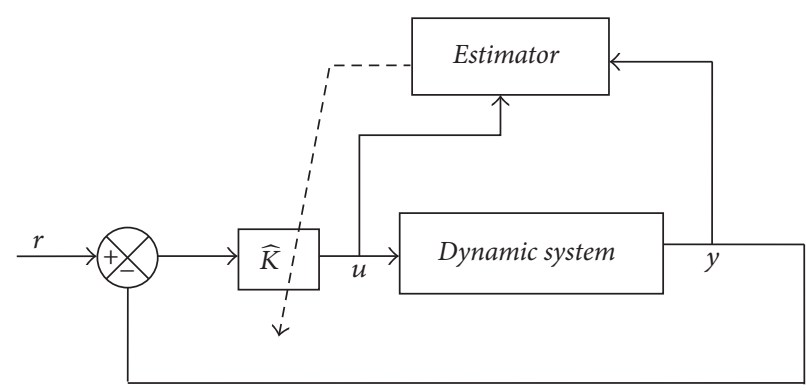

FIGURE 1: Block diagram for the proposed control system.

desired $y_{m}$. We used a continuous time formulation for the dynamical systems.

From (1)-(4) and Theorem presented above, we obtained the constant $\widehat{K}$ that determines the proportionality constant of the optimal control function $u$, given by (1). This development can be extended to any odd power of the control function $u$. Thus, if $n$ is an odd number, we replace (1) and (2):

$$
u=(K(r-y))^{n}
$$

Isolating $r$ produces

$$
\begin{aligned}
& \sqrt[n]{u}=K(r-y) \\
& r_{K}=\frac{\sqrt[n]{u}}{K}+y
\end{aligned}
$$

Then (8) and (10) are rewritten as

$$
\begin{gathered}
u_{m}=w_{m} * \sqrt[n]{u} \\
y-w_{m} * r_{K}=y-w_{m} *\left(\frac{\sqrt[n]{u}}{K}+y\right),
\end{gathered}
$$

respectively. The other equations are not modified.

The controller identification technique can be translated to the following procedure.

Step 1. Define the space-state from the dynamical system.

Step 2. Obtain the experimental data of the system $y(t)$; that is, define $y(0)=y_{0}$ and choose the desired trajectories $r$ and initial $K$.

Step 3. Integrate system (10)-(12).

Step 4. Find $\widehat{K}$.
Step 5. Update the input of the system $y(t)$ with results obtained in the previous step. Go to Step 3.

Step 6. After reaching the number of iterations previously stipulated to update $\widehat{K}$, update it and go to Step 2 .

Step 7. The simulation ends when the number of iterations has been reached.

Figure 1 shows a block diagram of the proposed control.

\section{Control of Chaos Using Controller Identification}

Simulation results are considered for three nonlinear dynamical systems: Rössler, Lotka-Volterra predator-prey for two preys and one predator, and Rössler hyperchaos, all systems being dimensionless. We consider independent control laws for each equation of the system. In controlled dynamic systems, the most common way to write the control function $\mathbf{u}=\mathbf{K}(\mathbf{r}-\mathbf{y})$ is given by

$$
u_{j}=K_{j}\left(\tilde{x}_{j}-x_{j}\right), \quad j=\{1,2, \ldots, n\}
$$

where $n$ is equal to the number of system equations. The references are $r=\tilde{x}_{j}$ and the entries are $y=x_{j}$. In [13], the control laws present proportional coefficients, but the equation is not independent because $u$ depends on all variables.

The software Matlab and Runge-Kutta of fourth order were used in the simulations. For the two systems, the values obtained from (6)-(12) and the state $y$ were computed concomitantly. A vector $Y$ was created to be used in the integration process of the Runge-Kutta function, defined as

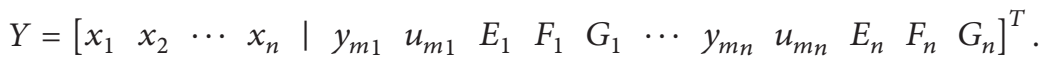

The initial state is given by

$$
Y(0)=Y_{0} \text {, }
$$

and the initial values $K_{j}$ are

$$
K_{j}=\left[\begin{array}{llll}
K_{1} & K_{2} & \cdots & K_{n}
\end{array}\right]^{T} .
$$


The transfer function $W_{m}(s)$ is

$$
W_{m}(s)=\frac{1}{s+1}
$$

In each case below, the reference to the system, containing the desired trajectory, will be represented by a vector $\tilde{x}_{j}$ as follows:

$$
\tilde{x}_{j}=\left[\begin{array}{llll}
\tilde{x}_{1}(t) & \tilde{x}_{2}(t) & \cdots & \tilde{x}_{n}(t)
\end{array}\right]^{T} .
$$

3.1. Local Controllability. Nonlinear systems can be written using direct parameterization to bring the nonlinear dynamics to the state-dependent coefficient (SDC) form

$$
\dot{\mathbf{x}}=\mathbf{A}(x) \mathbf{x}+\mathbf{B u},
$$

where $\mathbf{A}(x)$ is a state-dependent matrix. The design procedure consists of using direct parameterization of $\mathbf{A}(x)$ to bring the nonlinear system to a linear structure having statedependent coefficients (SDC) $f(x)=\mathbf{A}(x) \mathbf{x}$. In general, $\mathbf{A}(x)$ is unique only if $x$ is scalar. Then, many possibilities exist for SDC parameterizations if $x$ is not scalar.

The proposal is to show that the pair $[\mathbf{A}(x), \mathbf{B}]$ is a controllable parameterization of the nonlinear system (25) in a region where $K$ is updated, such that $[\mathbf{A}(x), \mathbf{B}]$ is pointwise controllable in the linear sense.

For each update of $K$, we consider the local controllability of the system. This idea is similar to locally optimal control from the state-dependent Riccati equation control $[18,19]$. In the two cases that follow, we will show that the control technique, proposed here for the nonlinear dynamical systems, produces systems that are locally completely state controllable.

3.2. Case 1: Application to the Control of the Rössler System. Rössler system [8] is given by

$$
\begin{aligned}
& \dot{x}_{1}=-x_{2}-x_{3}, \\
& \dot{x}_{2}=x_{1}+b x_{2}, \\
& \dot{x}_{3}=b+x_{3}\left(x_{1}-a\right) .
\end{aligned}
$$

Differential equations (26) define a continuous time system with chaotic behavior for $b=0.2$ and $a=5.7$. Notice that the third equation presents a nonlinearity $x_{3} x_{1}$. An interesting aspect of this system is its complex dynamical behavior in contrast with the simplicity of the description of its vector field. The phase portrait of Rössler system is shown in [8].

The system with control can be written as [13]

$$
\begin{aligned}
& \dot{x}_{1}=-x_{2}-x_{3}+u_{1}, \\
& \dot{x}_{2}=x_{1}+b x_{2}+u_{2}, \\
& \dot{x}_{3}=b+x_{3}\left(x_{1}-a\right)+u_{3} .
\end{aligned}
$$

Here, the objective of control strategy $u_{j}$ is to drive system (27) from any initial state $x_{j 0}$ to desired trajectories $\tilde{x}_{j}$. The desired trajectories are given by

$$
\tilde{x}_{j}=\left[\begin{array}{lll}
5+\cos (t) & 1+\sin (t) & 1+\sin (t)
\end{array}\right]^{T},
$$

which represents a limit cycle in the phase diagram.

The initial values for $K_{1}, K_{2}$, and $K_{3}$ were

$$
K_{j}=\left[\begin{array}{lll}
10 & 10 & 10
\end{array}\right]^{T}
$$

In order to obtain the simulation results, the values of $K_{1}$, $K_{2}$, and $K_{3}$ were periodically updated on each 0.5 from 0 to 100 .

Figure 2 presents the desired trajectories $r_{1}, r_{2}, r_{3}$ and the trajectories of the controlled system (27).

One can observe convergence with a residual error in the third component $x_{3}$. Figure 3 presents the phase portrait for the controlled Rössler system.

From Figure 3 we can notice convergence to the limit cycle given by $\tilde{x}_{j}$.

The local controllability for the Rössler system (27) can be obtained by rewriting the system in the state-dependent coefficients form (25). One possible parameterization for (27) is

$$
\dot{\mathbf{x}}=\left[\begin{array}{ccc}
0 & -1 & -1 \\
1 & b & 0 \\
\frac{b}{x_{1}} & 0 & x_{1}-a
\end{array}\right]\left[\begin{array}{l}
x_{1} \\
x_{2} \\
x_{3}
\end{array}\right]+\left[\begin{array}{lll}
1 & 0 & 0 \\
0 & 1 & 0 \\
0 & 0 & 1
\end{array}\right]\left[\begin{array}{l}
u_{1} \\
u_{2} \\
u_{3}
\end{array}\right]
$$

for $x_{1} \neq 0$.

The rank of the controllability matrix is computed for each update of $K$. It has value of 3 , which means that the nonlinear system (27) is locally completely state controllable.

3.3. Case 2: Application to the Control of the Biological Lotka-Volterra System. The biological Lotka-Volterra model describes populations in competition [11]. The general form of this model is given by

$$
\dot{x}_{j}=x_{j}\left(c_{j}-\sum_{j=1}^{n} a_{j i} x_{i}\right), \quad j=1,2,3
$$

where $x_{j}$ is density of the species $j$ at time $t, n$ is the number of species interacting in the system, $c_{j}$ is the reproduction or mortality rate, and $a_{j i}$ are predation, competition, or conversion rates. For two preys and one predator, or the two hosts and one parasitoid, the model becomes

$$
\begin{aligned}
& \dot{x}_{1}=x_{1}\left(c_{1}-a_{11} x_{1}-a_{12} x_{2}-a_{13} x_{3}\right), \\
& \dot{x}_{2}=x_{2}\left(c_{2}-a_{21} x_{1}-a_{22} x_{2}-a_{23} x_{3}\right), \\
& \dot{x}_{3}=x_{3}\left(c_{3}-a_{31} x_{1}-a_{32} x_{2}-a_{33} x_{3}\right) .
\end{aligned}
$$



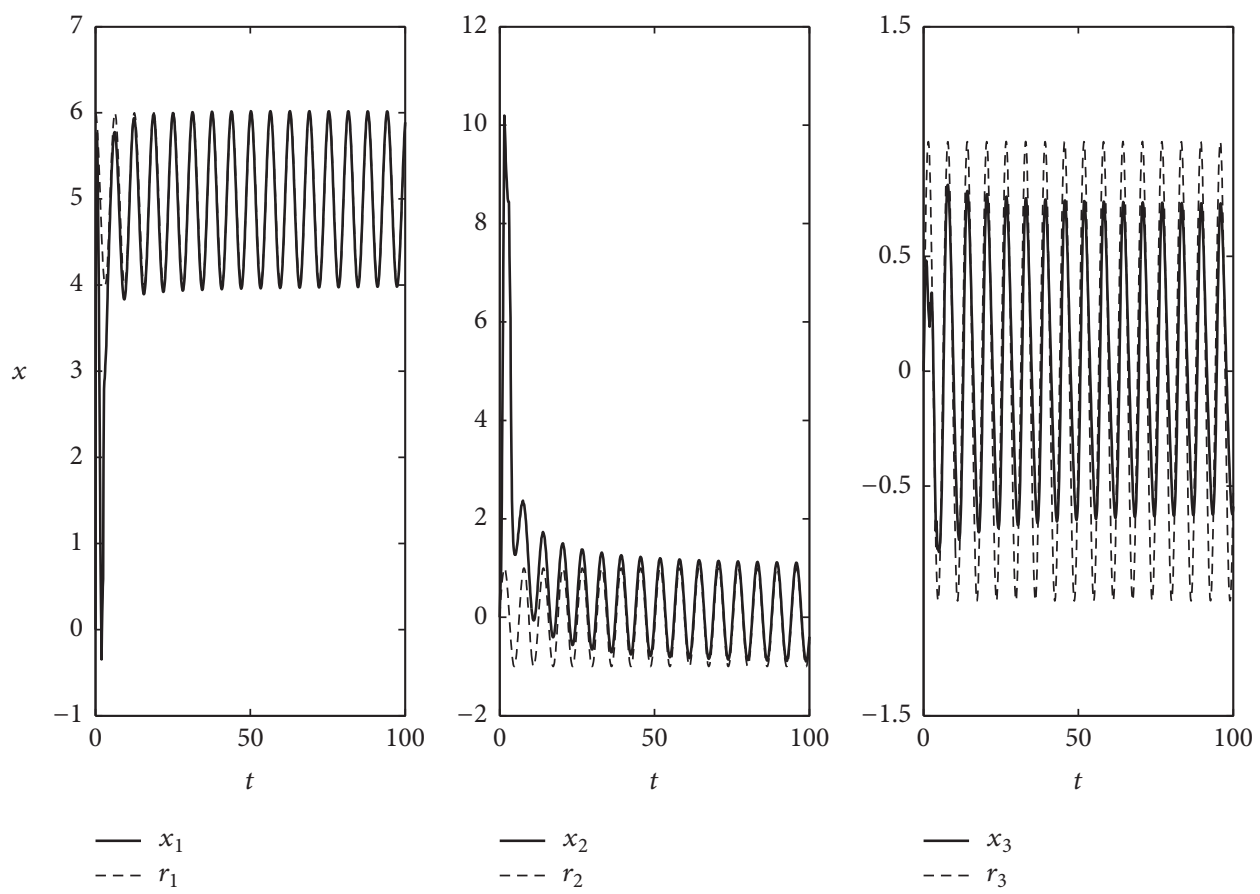

FIGURE 2: Temporal trajectories of the controlled system (27) and reference (28).

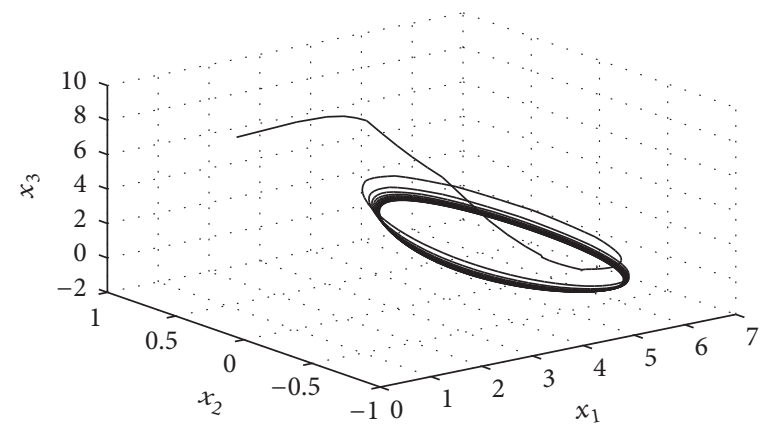

FIgURE 3: Phase portrait for the controlled Rössler system.

According to Gilpin [9] and Vance [10], system (32) is nonlinear and exhibits chaotic behavior for the following model coefficients: $c_{1}=c_{2}=-c_{3}=1, a_{11}=a_{12}=0.001$, $a_{22}=0.001, a_{21}=0.0015, a_{13}=0.01, a_{23}=0.001, a_{31}=$ $-0.005, a_{32}=-0.0005$, and $a_{33}=0$. The trajectories and the strange attractor of system (31) are shown in [16].

The controlled Lotka-Volterra system proposed is described by

$$
\begin{aligned}
& \dot{x}_{1}=x_{1}\left(c_{1}-a_{11} x_{1}-a_{12} x_{2}-a_{13} x_{3}\right)+u_{1}, \\
& \dot{x}_{2}=x_{2}\left(c_{2}-a_{21} x_{1}-a_{22} x_{2}-a_{23} x_{3}\right)+u_{2}, \\
& \dot{x}_{3}=x_{3}\left(c_{3}-a_{31} x_{1}-a_{32} x_{2}-a_{33} x_{3}\right)+u_{3} .
\end{aligned}
$$

The objective of control strategy $u_{j}$ is to drive system (33) from its initial state

$$
x_{j}(0)=x_{j 0}
$$

to the desired fixed point [20], or constant trajectories

$$
\tilde{x}_{j}=\left[\begin{array}{lll}
4 & 10 & 5
\end{array}\right]^{T},
$$

while minimizing performance criterion (11).

The initial values for $K_{1}, K_{2}$, and $K_{3}$ were the same as in Case 1 . The total simulation time was of 100 with $K_{1}, K_{2}$, and $K_{3}$ update on each 0.5 .

Figure 4 shows the time series of the controlled Lotka-Volterra system with initial densities $(2,5,2)$.

From Figure 4, one can observe that the trajectories are practically controlled when $t=20$. Figure 5 shows the phase portrait behavior of the controlled Lotka-Volterra system (33).

The performance index for the controllers change of the controlled Lotka-Volterra system is shown in Figure 6.

One can observe from Figure 6 that performance index has minimal variation after 100 (or constant trajectory), which leads us to conclude that it stabilizes at this value. 

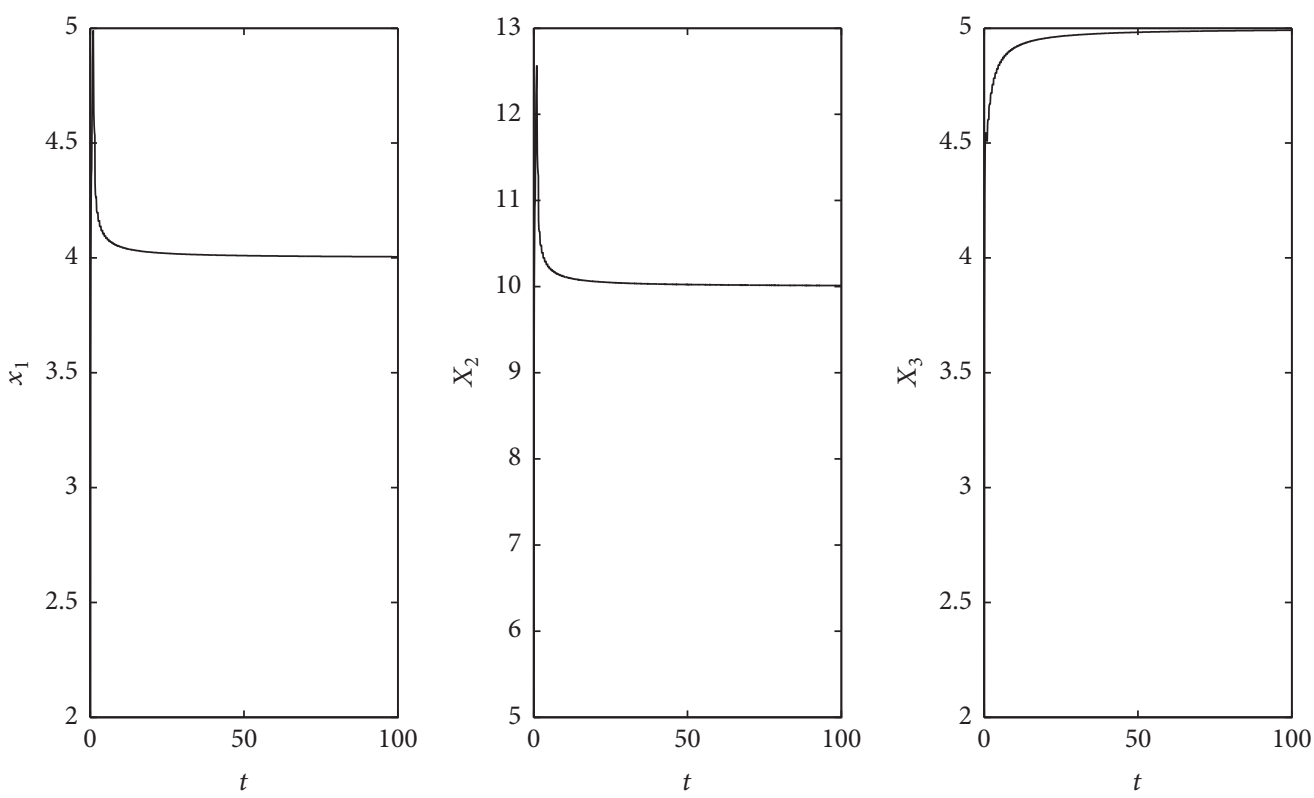

FIgURE 4: Temporal evolution of the controlled Lotka-Volterra system.

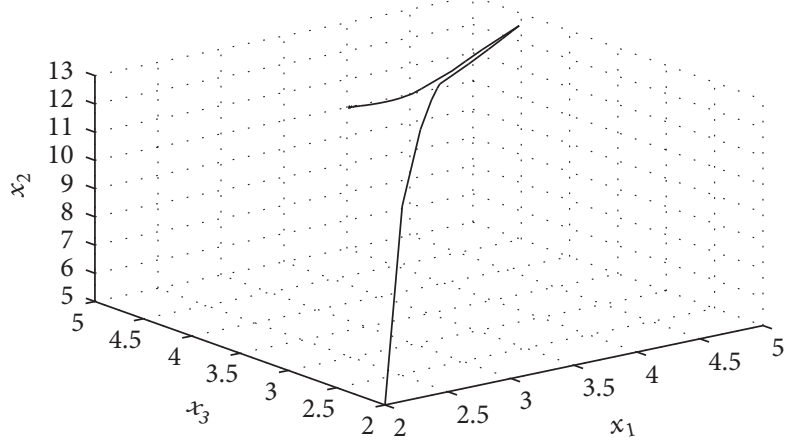

FIgURE 5: Phase portrait behavior of the controlled Lotka-Volterra system.
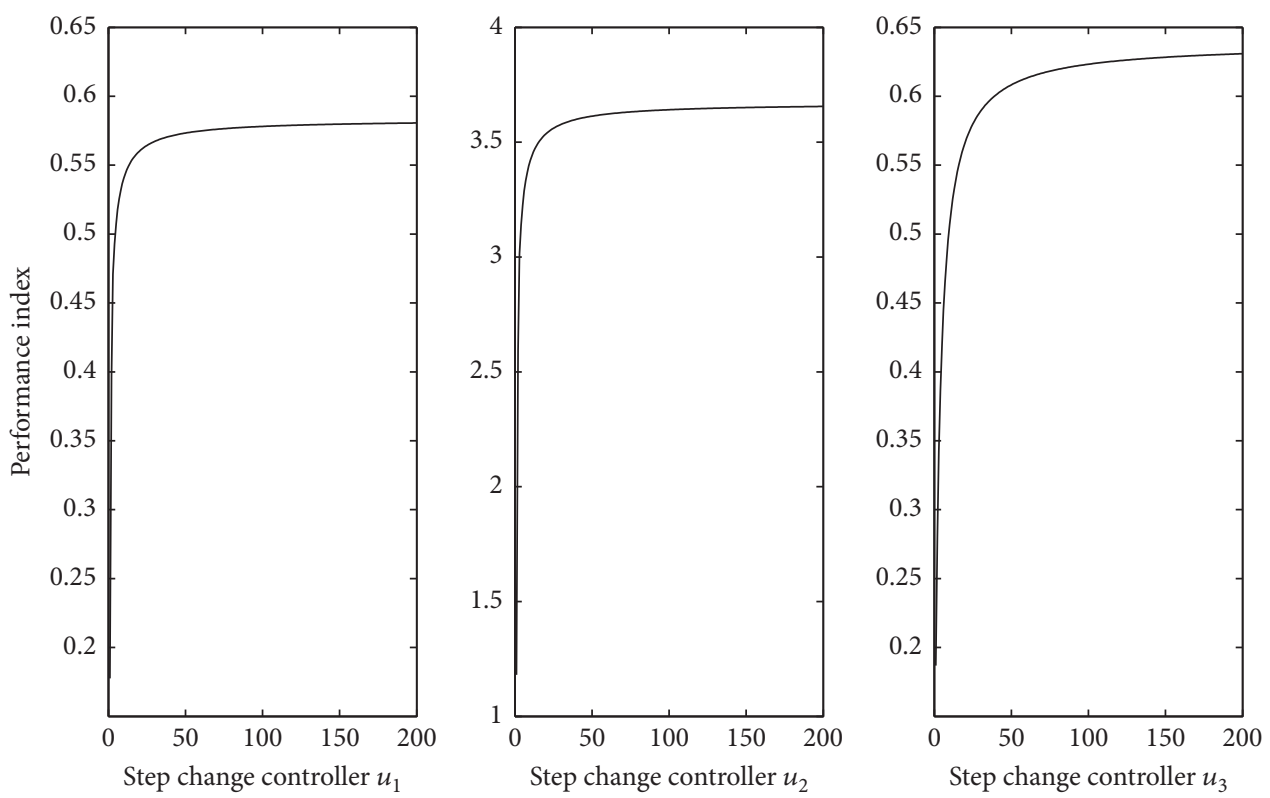

FIgURE 6: Performance index given by (11). 

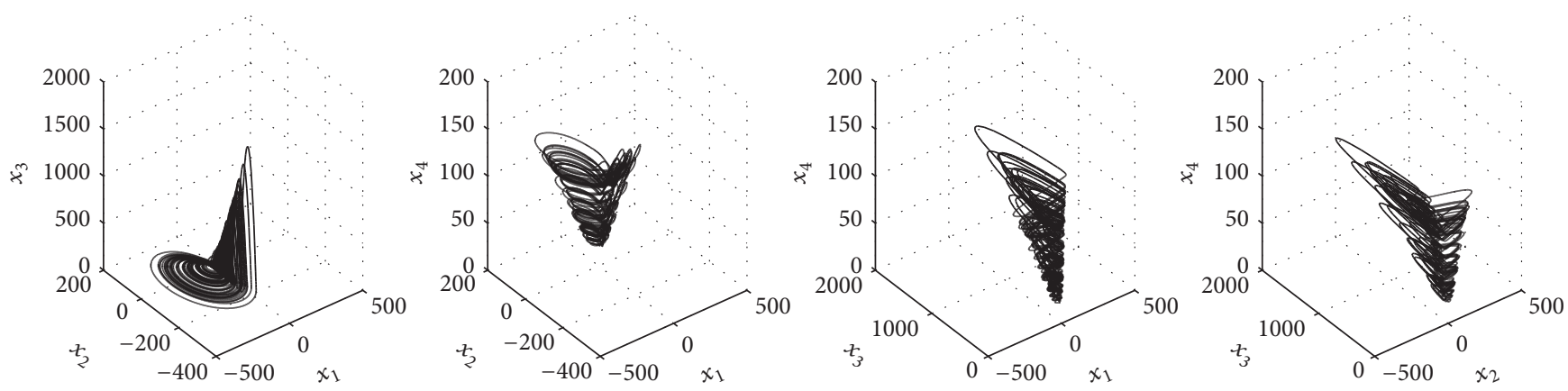

FIgURE 7: Phase portrait for the 4D Rössler system, with initial conditions $(0,0,0,30)$.

The local controllability for the Lotka-Volterra system (33) can be checked by rewriting the system in the statedependent coefficients form (25), parameterized by

$$
\begin{aligned}
\dot{\mathbf{x}}= & {\left[\begin{array}{ccc}
c_{1}-a_{11} x_{1} & -a_{12} x_{1} & -a_{13} x_{1} \\
-a_{21} x_{2} & c_{2}-a_{22} x_{2} & -a_{23} x_{2} \\
-a_{31} x_{3} & -a_{32} x_{3} & c_{3}-a_{33} x_{3}
\end{array}\right]\left[\begin{array}{l}
x_{1} \\
x_{2} \\
x_{3}
\end{array}\right] } \\
& +\left[\begin{array}{lll}
1 & 0 & 0 \\
0 & 1 & 0 \\
0 & 0 & 1
\end{array}\right]\left[\begin{array}{l}
u_{1} \\
u_{2} \\
u_{3}
\end{array}\right] .
\end{aligned}
$$

The rank of the controllability matrix is computed for each update of $K$. It has value 3 , which means that the nonlinear system (33) is locally completely state controllable.

3.4. Case 3: Application to the Control of the Rössler Hyperchaos System. Rössler hyperchaos system [12] is given by

$$
\begin{aligned}
& \dot{x}_{1}=-x_{2}-x_{3}, \\
& \dot{x}_{2}=x_{1}+b_{1} x_{2}+x_{4}, \\
& \dot{x}_{3}=b_{2}+x_{1} x_{3}, \\
& \dot{x}_{4}=-b_{3} x_{3}+b_{4} x_{4} .
\end{aligned}
$$

The $4 \mathrm{D}$ differential equations (37) define a continuous time system with chaotic behavior for $b_{1}=0.27857, b_{2}=3.0$, $b_{3}=0.3$, and $b_{4}=0.05$. As in the Rössler 3D system, the third equation presents a nonlinearity $x_{1} x_{3}$. The phase portraits of system (37) are shown in Figure 7, in four different combinations of coordinate axes.

The $4 \mathrm{D}$ system (37) with control can be written as

$$
\begin{aligned}
& \dot{x}_{1}=-x_{2}-x_{3}+u_{1}, \\
& \dot{x}_{2}=x_{1}+b_{1} x_{2}+x_{4}+u_{2}, \\
& \dot{x}_{3}=b_{2}+x_{1} x_{3}+u_{3}, \\
& \dot{x}_{4}=-b_{3} x_{3}+b_{4} x_{4}+u_{4} .
\end{aligned}
$$

Analogously to the previous cases, the control strategy $u_{j}$ drives system (38) from any initial state $x_{j 0}$ to desired trajectories $\widetilde{x}_{j}$. The desired trajectories are given by

$$
\tilde{x}_{j}=\left[\begin{array}{llll}
5+\cos (t) & \sin (t) & \sin (t) & 3+\sin (t)
\end{array}\right]^{T} .
$$

The initial values for $K_{1}, K_{2}, K_{3}$, and $K_{4}$ were

$$
K_{j}=\left[\begin{array}{llll}
0.0001 & 0.0001 & 0.0001 & 0.0001
\end{array}\right]^{T} .
$$

In order to obtain the simulation results, the values of $K_{j}$ were periodically updated on each 0.1 from 0 to 80 .

Figure 8 presents the desired trajectories $r_{1}, r_{2}, r_{3}, r_{4}$ and the trajectories of the controlled system (38).

One can observe in Figure 8 that there is convergence of the trajectories to the desired references.

The local controllability for the 4D Rössler system (38) can be obtained by rewriting the system in the statedependent coefficients form (25). As in the previous cases, the rank of the controllability matrix was computed for each update of $K$ as having value 4 , which means that the nonlinear system (38) is also locally completely state controllable.

Using the controller identification technique in the systems with chaotic behavior requires particular care in the choices of the initial conditions and the initial values of $K$. Although not shown in this work, several tests were performed with different choices of initial conditions and initial $K$. Each change in choices generates different control performances. Therefore, it is up to the control designer to analyze these initial data in order to better control the system.

\section{Conclusions}

In this paper we presented the controller identification technique applied to find proportional controllers for nonlinear systems with chaotic behavior. Simulation results were presented to illustrate the effectiveness of the technique. We observe convergence to the desired trajectories.

From the simulation results obtained, one can note that the controller identification technique is useful for the control of chaotic systems. Nevertheless, the results are dependent on the initial parameters $K$ and on the periodicity of the updating of $K$, which may indicate that the technique is not so robust in spite of its simplicity. In addition, we 

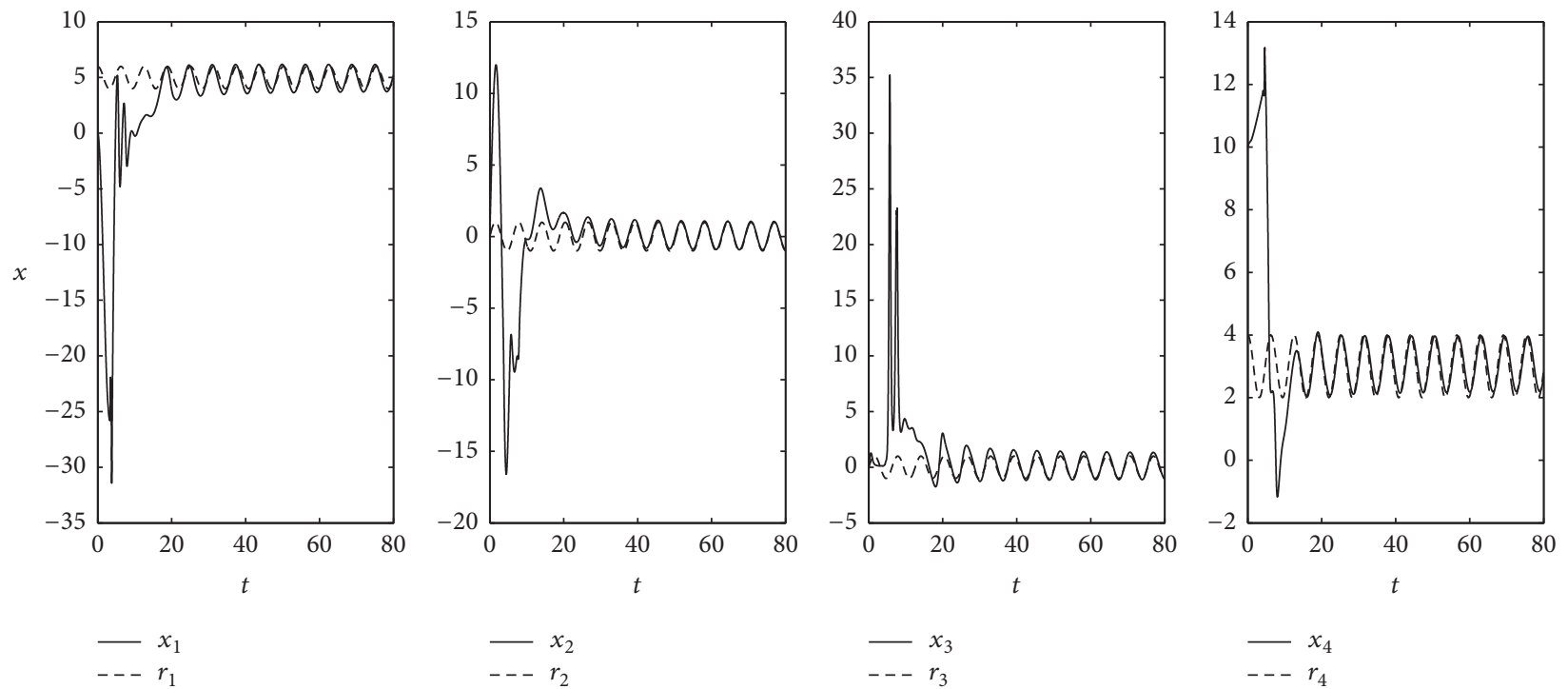

Figure 8: Temporal trajectories of the controlled system (38) and reference (39) with initial conditions $(0,0,0,10)$.

noted also that the controller for each system has its own peculiarities, which needs to be taken into account in each simulation. It is possible that one cannot find a general proof that the controller identification technique works for any nonlinear systems. However by the simplicity of application of the technique, we expect that it can be successfully used in other cases. It was shown in the simulations that the proposed control technique controls nonlinear systems that were locally completely state controllable.

Trying to improve the performance of the systems presented, higher order candidate controllers were used, but the performance was not improved. This leads us to believe that the results for the linear case represent a good illustration of the technique. Moreover, through the use of a simple control law, we could present the use of the technique in an illustrative way.

\section{Conflicts of Interest}

The authors declare that there are no conflicts of interest regarding the publication of this paper.

\section{References}

[1] M. G. Safonov and T.-C. Tsao, "The unfalsified control concept: a direct path from experiment to controller," Feedback Control, Nonlinear Systems, and Complexity, vol. 202, pp. 196-214, 1995.

[2] M. G. Safonov and T.-C. Tsao, "The unfalsified control concept and learning," Institute of Electrical and Electronics Engineers Transactions on Automatic Control, vol. 42, no. 6, pp. 843-847, 1997.

[3] J. M. Krause and P. P. Khargonekar, "A comparison of classical stochastic estimation and deterministic robust estimation," Institute of Electrical and Electronics Engineers Transactions on Automatic Control, vol. 37, no. 7, pp. 994-1000, 1992.

[4] K. Poolla, P. Khargonekar, A. Tikku, J. Krause, and K. Nagpal, "A time-domain approach to model validation," Institute of
Electrical and Electronics Engineers Transactions on Automatic Control, vol. 39, no. 5, pp. 951-959, 1994.

[5] R. S. Smith and J. C. Doyle, "Model validation: a connection between robust control and identification," Institute of Electrical and Electronics Engineers Transactions on Automatic Control, vol. 37, no. 7, pp. 942-952, 1992.

[6] M. G. Safonov and F. B. Cabral, "Fitting controllers to data," Systems \& Control Letters, vol. 43, no. 4, pp. 299-308, 2001.

[7] M. G. Safonov, "Origins of robust control: Early history and future speculations," in Proceedings of the 7th IFAC Symposium on Robust Control Design, ROCOND'12, pp. 1-8, June 2012.

[8] O. E. Rössler, "An equation for continuous chaos," Physics Letters A, vol. 57, no. 5, pp. 397-398, 1976.

[9] M. E. Gilpin, "Spiral chaos in a predator-prey model," The American Naturalist, vol. 113, no. 2, pp. 306-308, 1979.

[10] R. R. Vence, "Predation and resource partitioning in one predator-two prey model communications," The American Naturalist, vol. 112, no. 987, pp. 797-813, 1978.

[11] V. Volterra, Leçons sur la Théorie Mathematique de la Lutte pour la Vie. Gauthier-Villars, Paris, 1931.

[12] R. Barrio, M. A. Martínez, S. Serrano, and D. Wilczak, "When chaos meets hyperchaos: 4D Rössler model," Physics Letters A, vol. 379, no. 38, pp. 2300-2305, 2015.

[13] M. Rafikov and J. M. Balthazar, "On control and synchronization in chaotic and hyperchaotic systems via linear feedback control," Communications in Nonlinear Science and Numerical Simulation, vol. 13, no. 7, pp. 1246-1255, 2008.

[14] J. Sun and Y. Zhang, "Impulsive control of Rössler systems," Physics Letters A, vol. 306, no. 5-6, pp. 306-312, 2003.

[15] A. El-Gohary and M. T. Yassen, "Optimal control and synchronization of Lotka-Volterra model," Chaos, Solitons \& Fractals, vol. 12, no. 11, pp. 2087-2093, 2001.

[16] A. Molter and M. Rafikov, "Nonlinear optimal control of population systems: applications in ecosystems," Nonlinear Dynamics, vol. 76, no. 2, pp. 1141-1150, 2014.

[17] M. Rafikov, J. M. Balthazar, and H. F. von Bremen, "Mathematical modeling and control of population systems: applications in 
biological pest control," Applied Mathematics and Computation, vol. 200, no. 2, pp. 557-573, 2008.

[18] H. T. Banks, B. M. Lewis, and H. T. Tran, "Nonlinear feedback controllers and compensators: A state-dependent Riccati equation approach," Computational Optimization and Applications, vol. 37, no. 2, pp. 177-218, 2007.

[19] C. P. Mracek and J. R. Cloutier, "Control designs for the nonlinear benchmark problem via the state-dependent Riccati equation method," International Journal of Robust and Nonlinear Control, vol. 8, no. 4-5, pp. 401-433, 1998.

[20] A. Molter and M. Rafikov, "Controle ótimo para um Sistema Caótico de Lotka-Volterra," TEMA - Tendências em Matemática Aplicada e Computacional, vol. 5, no. 2, 2004. 


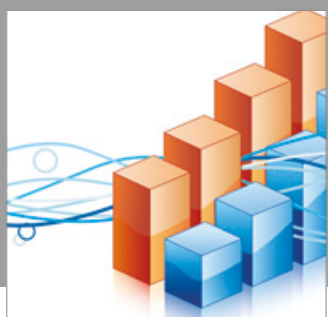

Advances in

Operations Research

vatersals

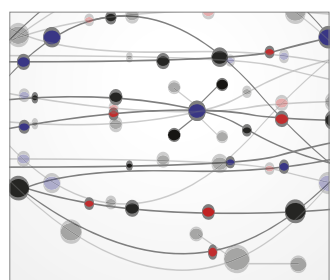

\section{The Scientific} World Journal
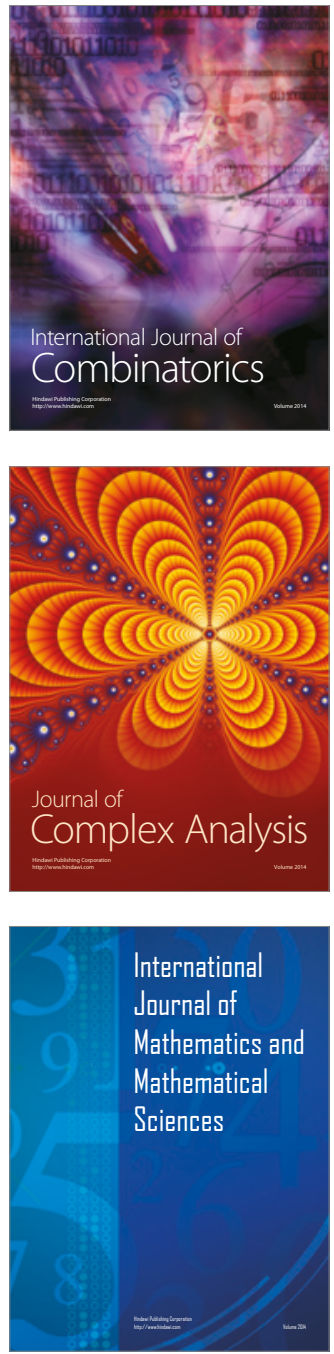
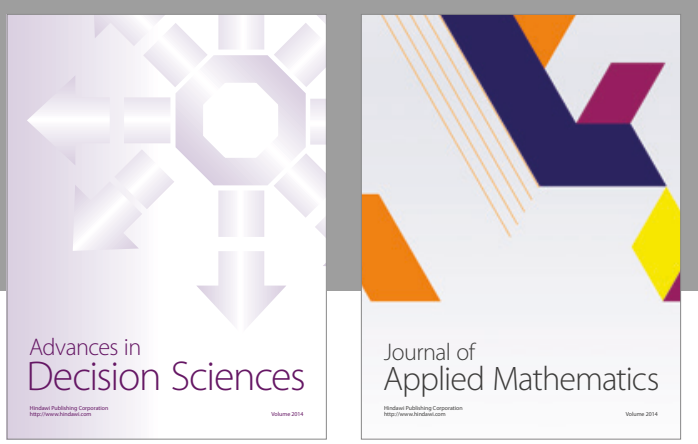

Algebra

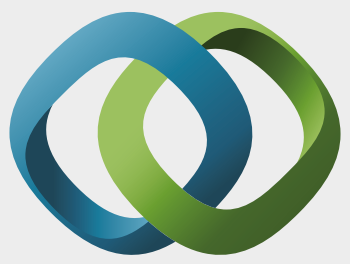

\section{Hindawi}

Submit your manuscripts at

https://www.hindawi.com
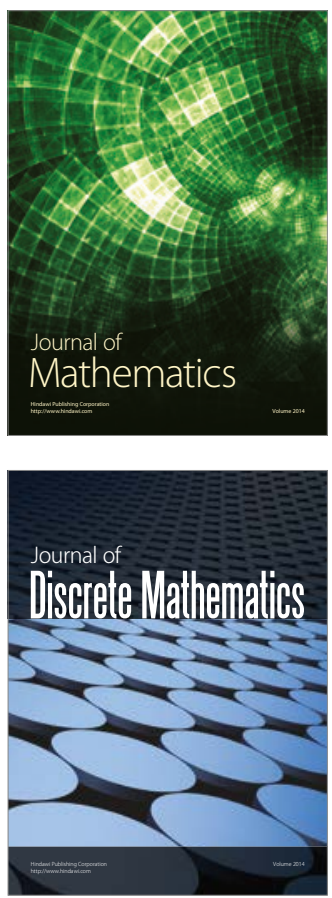

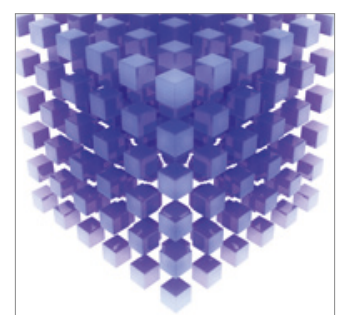

Mathematical Problems in Engineering
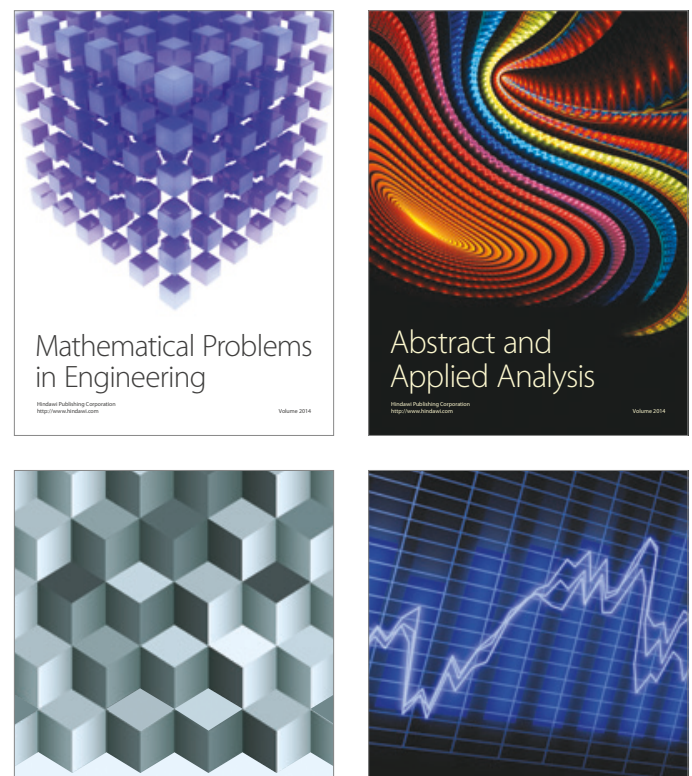

Journal of

Function Spaces

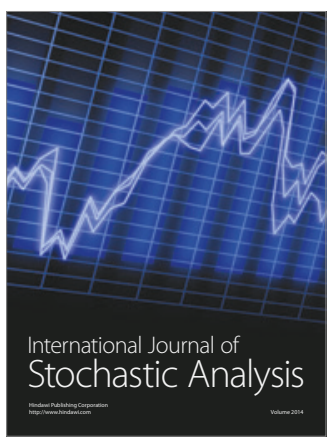

Probability and Statistics
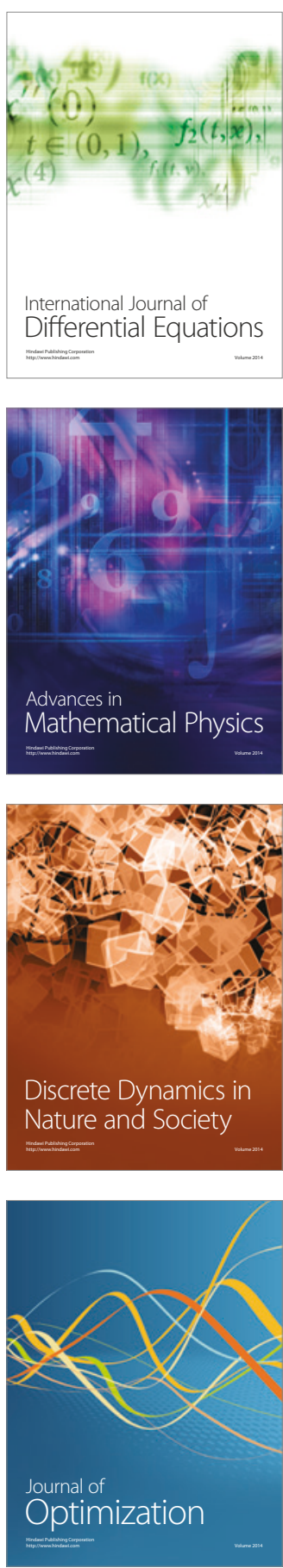